\title{
DROP IMPACT ON TEXTILE MATERIAL: EFFECT OF FABRIC PROPERTIES
}

\author{
Zouhaier Romdhani ${ }^{1 *}$, Ayda Baffoun², Mohamed Hamdaoui ${ }^{2}$, Sadok Roudesli' \\ ${ }^{1}$ Laboratory of Interfaces and Advanced Materials (L.A.M.I), faculty of sciences of Monastir - University of Monastir - Tunisia \\ ${ }^{2}$ Laboratory of thermal and energizing system studies, (L.E.S.T.E), National Engineering School of Monastir (E.N.I.M), University of Monastir - Tunisia \\ Avenue de l'Environnement 5019 Monastir Tunisie \\ *Corresponding author. Tel.: (+216) 295181 20; Fax: (+216) 735017 87, e-mail : romdhanizouhaier@gmail.com
}

\begin{abstract}
:
This paper presents an experimental study of impact of water drop on a surface in a spreading regime with no splashing. Three surfaces were studied: virgin glass, coating film and woven cotton fabric at different construction parameters. All experiments were carried out using water drop with the same free fall high. Digidrop with highresolution camera is used to measure the different parameters characterising this phenomenon. Results show an important effect of the height of the free fall on the drop profile and the spreading behaviour. An important drop deformation at the surface impact was observed. Then, fabric construction as the weft count deeply affects the drop impact. For plain weave, an increase of weft count causes a decrease in penetration and increase in the spreading rate. The same result was obtained for coated fabric. Therefore, the impact energy was modified and the drop shape was affected, which directly influenced the spreading rate.
\end{abstract}

\section{Keywords:}

Contact angle, Drop impact, Woven fabric, Drying process, Coating.

\section{Introduction}

Wetting and spreading phenomena on porous media are being investigated for various applications including inkjet printing. In this case, much progress in wetting has been made, thanks to improvements in contact angle measurements. However, some questions of drop spreading have remained unanswered. In this case, the contact angle is the key boundary condition in drop spreading. It is defined as the angle formed between the moving liquid interface and the solid surface at the line of the three-phase contact. It was originally defined in early 1800 s. It was been explained for non-porous and non-absorbent surfaces [5]. The equilibrium contact angle of liquid on an ideal solid surface follows the well-known Young's equation [18] shown by relation.

$$
\cos \theta_{Y}=\frac{\gamma_{s v}-\gamma_{s l}}{\gamma_{l v}}
$$

where the variable $y$ refers to interfacial tension of the solidliquid (sl), solid-vapour (sv) and solid-liquid interfaces (sl).

The influence of the liquid properties and the porous media parameters on the overall phenomenon was very significant. The static phenomenon was widely studied and many parameters characterising the liquid-surfaces interactions were studied $[2-4,15]$. Other researchers were interested in the study of the Young's equation on rough and heterogeneous surfaces [8-10].

In the case of the drop impacting or dynamic spreading on a solid substrate, it goes back up to 1732, where an absence of direct contact between the drop and the heated material was observed. However, the first real study was made by Leidenfrost [6]. The Leidenfrost effect consists in the quasi-instantaneous formation of a film steam under the drop, just before its contact with the heated substrate. It has been shown that the dynamic and thermal features of these impacts are influenced by many parameters, as shown in Figure 1. The interface interactions depend on liquid-phase properties, the physical characteristics of vapour and substrate. This phenomenon results in three outcomes: bouncing, spreading or splashing. Six possible scenarios of drop impact on a solid surface are deposition, prompt splash, corona splash, receding break-up, partial rebound and complete rebound [10].

Many factors characterising the liquid drop impact on a solid substrate phenomenon exist in literature and the most important are the Weber number, which corresponds to the report between the inertial forces and superficial tension of the drop; the Reynolds number, which defines the report between the inertial forces and the viscous forces; and the number Ohnesorge as a combination between the last two adimensional numbers (Reynolds and Weber). These parameters were given respectively by the following equations:

$$
\begin{array}{ll}
W e_{n}=\frac{\rho_{l} V_{i, n}^{\mathbf{2}} D_{0}}{\sigma_{l}} & e q(2) \\
R e_{n}=\frac{\rho_{l} V_{i, n} D_{0}}{\mu_{l}} & e q(3) \\
O \boldsymbol{h}=\frac{\sqrt{W e}}{R e}=\frac{\mathbf{1}}{\sqrt{L a}} & e q(4)
\end{array}
$$




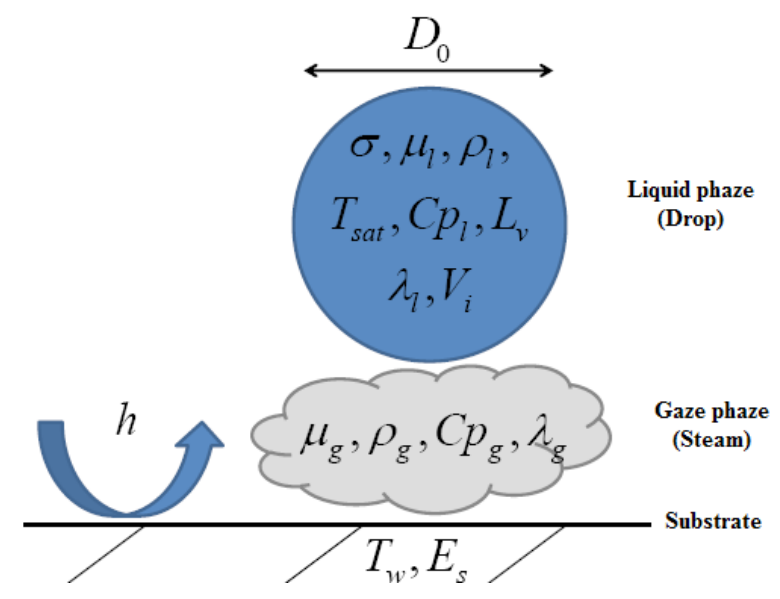

Figure 1. Dynamic and thermal features of drop impact on solid substrate. Where for the liquid phase, D0 is the drop diameter Vi is the initial speed initial, $\alpha i$ is the impact angle, $\mu \mathrm{l}$ is the viscosity of liquid, $\sigma \mathrm{l}$ is the superficial tension, $\mathrm{Cpl}$ is the thermal capacity, $\rho \mathrm{l}$ is the density, Tsat is the temperature of saturation. For the steam and solid substrate, $\mu \mathrm{g}$ is the steam viscosity, Cpg is the thermal capacity, $\rho g$ is the density, $\lambda$ is the coefficient of convective exchange between the substrate and the liquid, Tw is temperature of substrate and Lv is the latent heat of state change.

Where $\mathrm{Vi}, n, D 0$ and $\rho_{1}, \sigma_{1}$ are respectively the incidental normal speed of the drop, the diameter, the density of the liquid and the superficial tension of the liquid.

The liquid drop impact on solid substrate is a very complex phenomenon that depends on several factors as cited before. This phenomenon can be drawn by injection of a unique drop or by successive drops. In that setting, several cases can be considered as the drop impact on a cold substrate which corresponds to our study. This regime of drop-substrate impact is defined as the used liquid has a temperature lower than the boiling point, that is, $T^{*} \leq 0$, where $T^{*}$ was defined as given by equation.

$$
T^{*}=\frac{T_{w}-T_{b}}{T_{\text {Leid }}-T_{b}}
$$

$T_{b}$ is the boiling temperature of liquid and $T_{\text {Leid }}$ is the Leidenfrost temperature. This parameter is negative for temperature values lesser than boiling point, between 0 and 1 for temperatures between Teb and TLeid, and greater than 1 where the temperature is more than Leidenfrost value.

Several researchers are interested [7-9] and two regimes can be investigated: the drop deposit and the splashing. For the first state, the drop has a weak kinetic energy, which vanishes under viscosity effect. The drop falls, spreads and evaporates by natural convection. Second, as the kinetic energy of the drop increases, the viscous strength cannot dissipate the totality of the initial kinetic energy. This important energy leads to the drop splashing and the formation of secondary drop that will be subsequently set down on the substrate. For these states, the Weber, Ohnesorge or Reynolds numbers are used to define a unique factor as the Mundo [8] number, which is expressed by the following relation:

$$
K=O \boldsymbol{h} W e^{-0,4}
$$

In this domain, Worthington [20] studied more precisely the dynamics of the water drop impact, notably the different stages of the rebound of a drop on a hot substrate, which are shown in Table 1.

The literature presents several studies examining the phenomenon of drop impact on a heated substrate and various parameters have been introduced to characterize this state precisely in the case of rebound and splashing phenomena. The impact speed, drop size, liquid viscosity and surface tension also have an effect on the tendency of splashing $[11,12,19]$. Associated with these properties, more models of splashing threshold have been proposed $[13,14]$.

This work is in the context of dynamic spreading of droplets on a cold substrate. A glass slide and a textile support are used in

Table 1. Different regimes of drop impact on solid surface.

\begin{tabular}{|c|c|c|}
\hline $\begin{array}{c}\text { Temperature of } \\
\text { the substrate }\end{array}$ & $\begin{array}{c}\text { Mundo or Weber } \\
\text { number }\end{array}$ & Drop regime \\
\hline $0 \leq \mathrm{T}^{*} \leq 0,5$ & Independent & The deposition of the drop is observed, it is a wetting regime \\
\hline \multirow{2}{*}{$0,5 \leq \mathrm{T}^{*} \leq 1$} & $\mathrm{~K}<1000$ & The drop bounces off on the substrate \\
\cline { 2 - 3 } & $\mathrm{K}>1000$ & The regime splashing is observed \\
\hline \multirow{3}{*}{$\mathrm{T}^{*}>\mathrm{T}_{\text {Leid }}$} & $W e, n \leq 30$. & This is the rebound regime \\
\cline { 2 - 3 } & $30 \leq \mathrm{We}, \mathrm{n} \leq 70$ & First, a large deformation of the drop and the formation of some secondary drops \\
\cline { 2 - 3 } & We, $\mathrm{n} \geq 70$ & The presence of splashing phenomenon and the formation of several secondary \\
\end{tabular}


this experiment. First, we will look at the effect of the injection distance of the drop on the virgin glass. Secondly, the general behaviour of the drop impact was investigated. Then, the effect of the support parameters on the impact and spreading was studied. The results are based on the study of the variation of the contact angle, the height and the diameter of impact over time.

\section{Experimental}

\subsection{Glass Treatment}

Before experiment setting, the gill of glass has been cleaned to the ultrasonic sounds during 30 seconds in ethanol, then rinsed with the same solvent and dried in nitrogen. This cleaning process permits to remove the contamination organic contamination on the substrate surface. Then the surface is treated and activated in an oxidizing solution 'Piranha' at $50^{\circ} \mathrm{C}$ for $30 \mathrm{~min}$. This solution is a mixture of $3: 7 \mathrm{v} / \mathrm{v}$ of hydrogen peroxide $\left(\mathrm{H}_{2} \mathrm{O}_{2}\right)$ at $30 \%$ and concentrated sulphuric acid $\left(\mathrm{H}_{2} \mathrm{SO}_{4}\right)$. After this treatment the gill of glass is rinsed with twice-distilled water and then dried under flux of nitrogen. At this stage of treatment, the glass surface is enriched in hydroxyl $(-\mathrm{OH})$ or even covered with some film of water molecular (link hydrogen) and therefore becomes absorbent.

\subsection{Coating Substance}

The dispersive coating solution has been taken from a textile printing process specialised in ink-jet printing. Its formulation is shown in Table 2.

Table 2. Chemical composition of dispersive coating agent

\begin{tabular}{|c|c|}
\hline Chemical product & Value (\%) \\
\hline Setaprint RST-A (thickener) & 4 \\
\hline Setaprint NDG (oxidant) & 1.5 \\
\hline Urea (swelling agent) & 15 \\
\hline Sodium bicarbonate & 2.5 \\
\hline
\end{tabular}

\section{$\underline{2.3 \text { Coating Film Preparation }}$}

The film of the printing solution was set down by spin-coating on the gill of absorbent glass $\left(2.5 \times 2.5 \mathrm{~cm}^{2}\right)$. It is cleaned and treated as mentioned before, for 30 seconds at an angular velocity $2000 \mathrm{rpm}$.

\subsection{Drying Process}

The study of the drying morphology of the coating film was examined with two temperatures: the ambient laboratory environment $\left(25^{\circ} \mathrm{C}, 30 \% \mathrm{RH}\right)$ and the oven-drying process at $110{ }^{\circ} \mathrm{C}$ in 2 minutes.

\section{$\underline{2.5}$ Fabric Coating}

Experiments were carried out on virgin glass, uncoated and coated cotton woven fabrics. The weaving structure is plain weave; the warp count was 22 per $\mathrm{cm}$ and the weft count was varied as 11,17 and 22 per $\mathrm{cm}$. With regard to treating woven fabric, the reverse-rollers coating technique was used to apply the coating paste on the fabric.

\subsection{Spreading Kinetic Measurement}

The kinetic spreading parameters were studied using water droplets and the evolution of the drop profile was captured with a video camera of GBX Digidrop with 25 captures per second. The water volume used was $5 \mu \mathrm{l}$ and every measure was repeated 3 times.

\section{Results and discussions}

\subsection{General behaviour of drop impact}

In this part, the effect of the distance of drop fall was studied and the general behaviour of the drop impact on virgin glass and coating film were investigated. This film was dried in two processes at ambient-dried and oven-dried conditions. In all experiments, the drop injection is by free fall. Results were judged according to the drop profile, the contact angle variation, the variation of the adimensional diameter and the height during the time. First, the drop was allowed to fall from two distances, 5 and $10 \mathrm{~cm}$. The contact angle variation presents two phases: a drop deformation at first short time and spreading in the second phase. This deformation is very important for important used high. It is explained by the most important energy of the drop. It is potential energy which is directly bound to the height of the drop fall. But the difference between the two studied heights is not very important because of the weak mass of the drop. Therefore, the transformation of this energy into surface energy is uniquely responsible for the drop deformation at the first impact phase as shown in Figure 2 and the drop shape as in Figure 3.

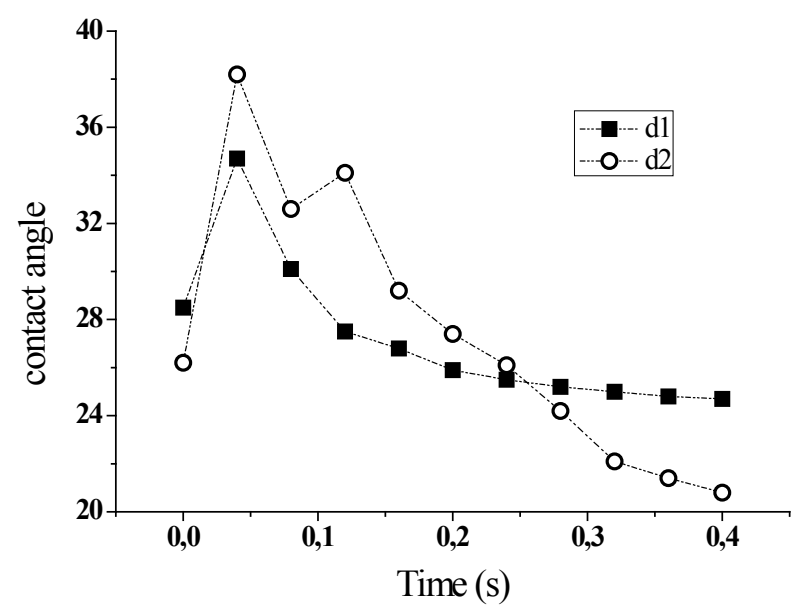

Figure 2. Effect of the distance of free fall on the contact angle variation

By comparison of different studied parameters of the drop impacting the deformation and the spreading phenomena were identified. The process of the drop impacting is divided into two phases: the initial phase of drop impact in very first short time and the second when the maximal drop spreading is reached. The first stage results from the fact that the drop 
partially takes off the surface, whereas it is again in a phase of vertical elongation as shown in drop shape in Figure 3 . It is a very important deformation in the vertical sense, contrary to the horizontal direction as illustrated in the contact angle, the diameter, and the height variation in Figures 4, 5 and 6.

This behaviour was due to the surface tension of the different systems used in the experiment. Therefore, the coated film in oven-drying conditions has an important energy which affects the impact phenomenon. Then, the presence of the air bubbles under the drop surface influences the drop deformation. This deformation is confirmed by the variation of the contact angle during the first $120 \mathrm{~ms}$. It is very important for the coating film dried in an oven-drying process.

In terms of energy, this deformation is the transformation of the total energy into surface energy. The energy of the drop before impact is transformed into surface energy during the deformation that also comes with the movement of the liquid inside the drop. Therefore, the internal flow possesses a kinetic energy that varies with the deformation and that is dissipated progressively because of the liquid viscosity. Conservation of the total energy in this system can be summarised as follows:

$$
\frac{d E}{d t}+\Pi_{\text {diss }}=\mathbf{0}
$$

Where $\Pi_{\text {diss }}$ is the dissipated power by the viscous effects and $E$ corresponds to the mechanical energy of the system in the absence of evaporation (the evaporation was disregarded because of the small drop size and short duration of spreading phenomenon):

$$
E=E_{c}+E_{p}+E_{c, \text { int }}+\sigma A \quad e q
$$

Where $E_{c}$ is the kinetic energy, $E_{c, \text { int }}$ is the kinetic energy of the internal drop flow, $E_{p}$ is the potential energy, $\sigma A$ is the surface energy

During the second phase, the drop spreads until all the initial energy is transformed into surface energy or is dissipated. Thus, when the maximal spreading is reached, all the mechanical energy meets under the shape of surface energy. Therefore, the conservation of energy is given by

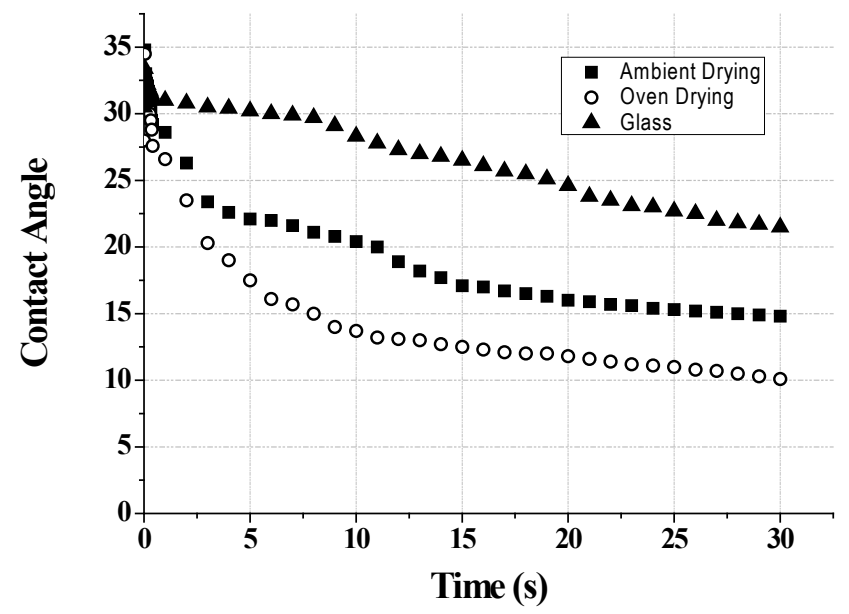

$$
E=\frac{A_{\max }}{A_{0}}+w_{\max }
$$

With $\mathrm{w}_{\max }$ : the dissipated energy during the spreading returned to $\sigma A_{0}$. In this Dans cette expression, $A_{0}$ and $A_{\max }$ correspond to the surfaces of the drop respectively in its initial spherical state and in its maximal deformation.

Variation in height (see figure) is divided into two categories that correspond to two phenomena: the drop deformation and the spreading. After drop stabilization, the strong reduction of the height in the first instants translated the predominance of the spreading and the total absence of evaporation in this interval. In these instants, the curve shows a spreading which has maximal speed and amplitude. To study the kinetic spreading of the couple drop/coating film, the notion of the reservoir imposes itself to explain the different obtained results. This space depends on the film thickness as well as its drying method. From Figures 4,5 and 6 , it can be seen that the spreading rate on the ambient-dried coatings, though much lower as compared to oven-dried coating, still remains slightly higher than on virgin glass. Therefore, it has the more important kinetic (Dt/D0) for the oven-dried film and oven-dried coted fabric. This result is confirmed by the adimensional height variation on the coated fabric that represented in Figure 6.
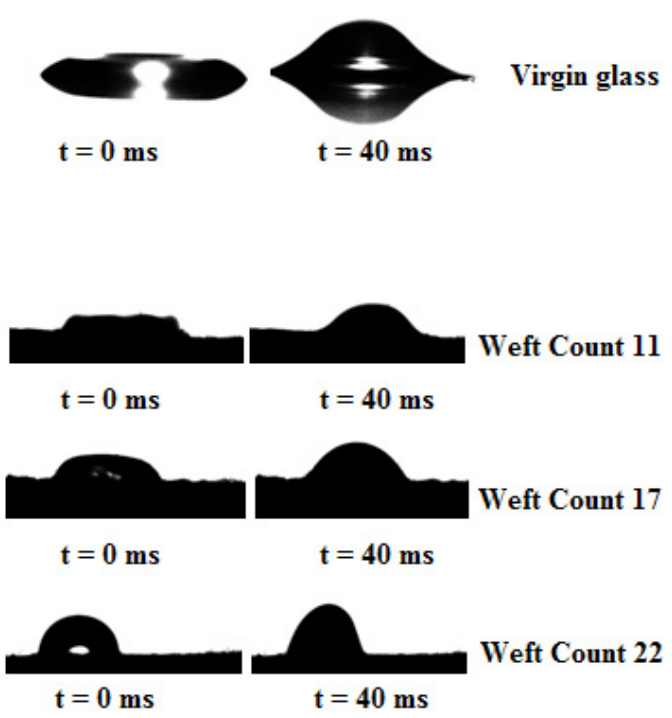

Figure 3. The variation of the drop shape just after impact

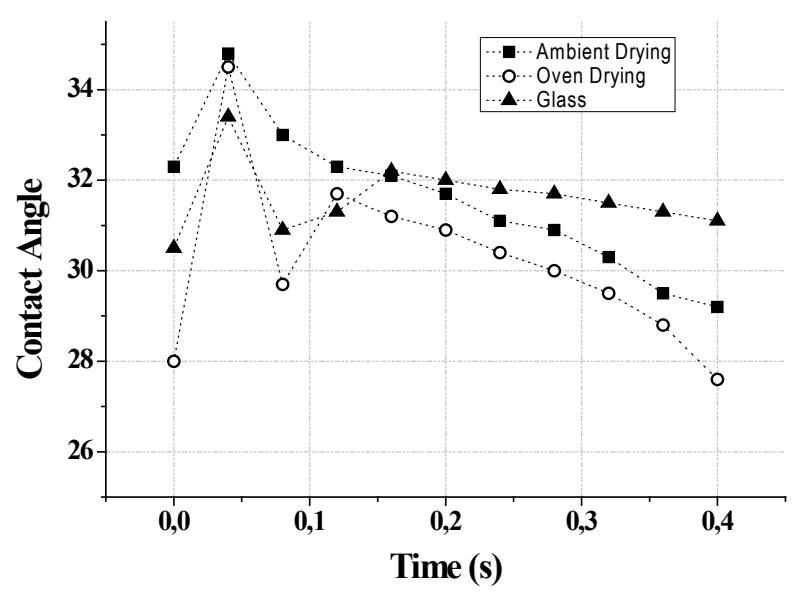

Figure 4. Contact angle variation on virgin glass and coating film dried in two drying process 

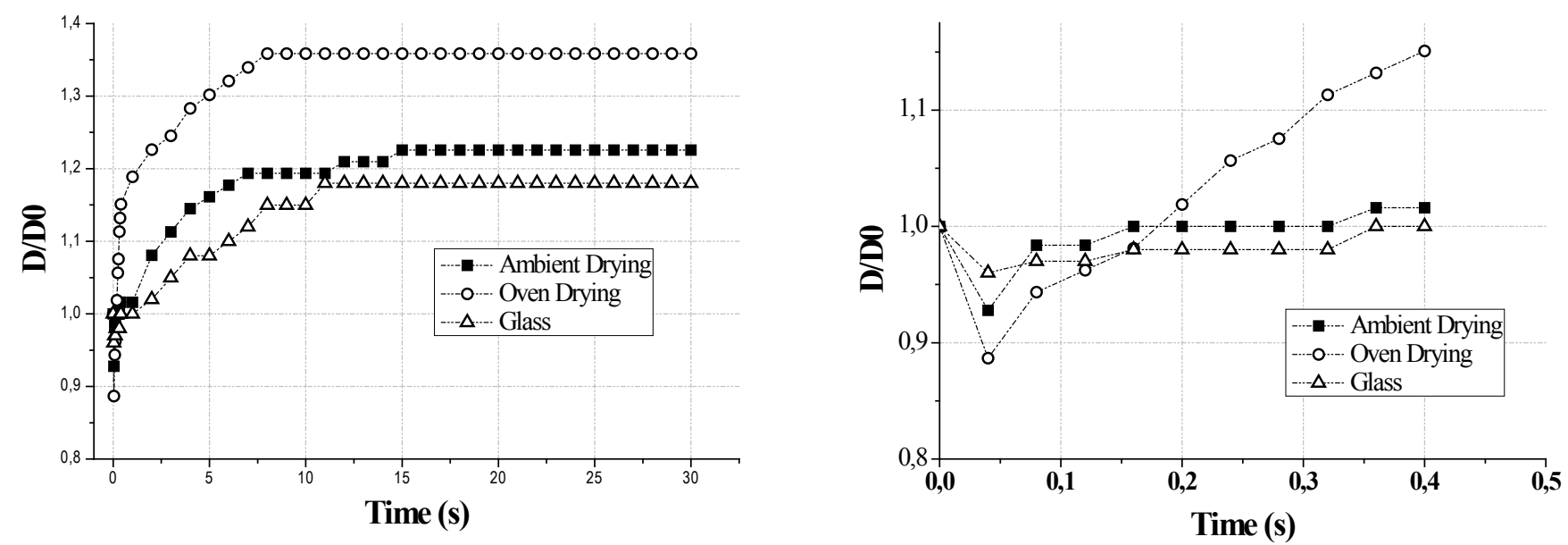

Figure 5. Adimensional diameter variation on virgin glass and coating film dried in two drying process
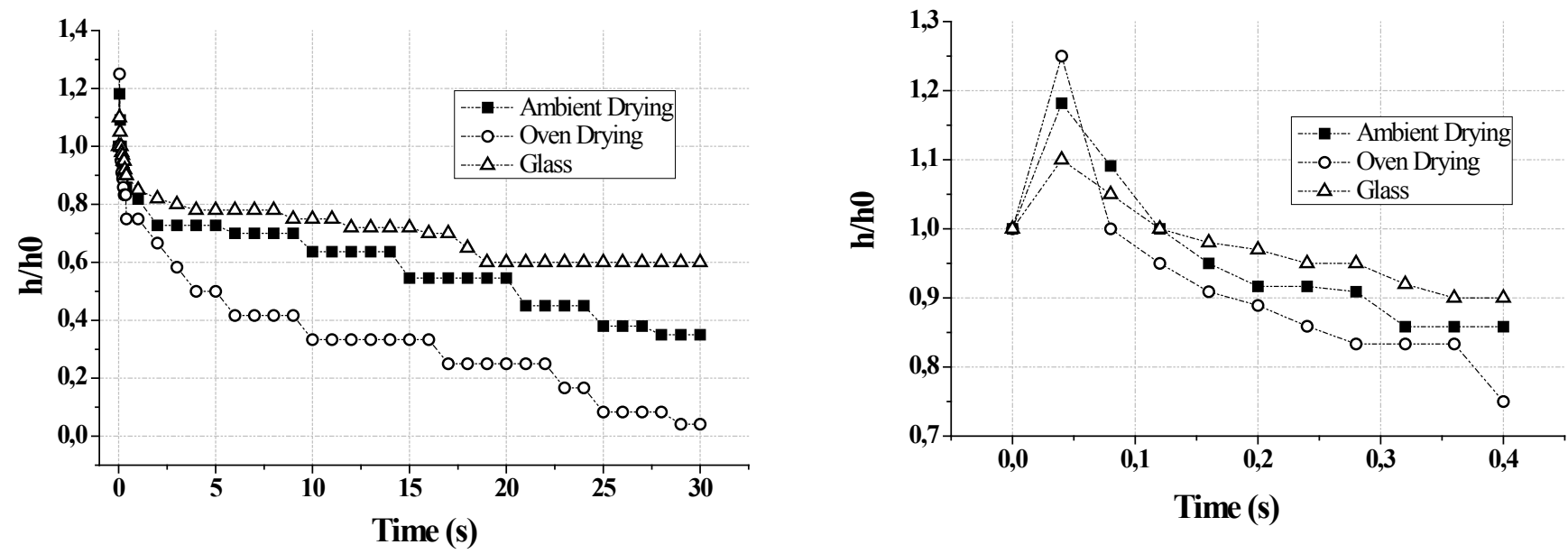

Figure 6. Adimensional height variation on virgin glass and coating film dried in two drying process

\subsection{Effect of fabric construction}

The drop impact phenomenon was studied on cotton woven fabric at three different weft counts and at the same height of drop fall. This phenomenon of water drop can be classified into three distinguishable stages: the first stage, in which the vertical deformation of drop profile as growing of the drop diameter as shown in Figure 3 . Then, the contact radius on the surface increases to its maximum and remains relatively constant over a period of time. Finally, the penetration where the contact radius shrinks and the liquid drop is absorbed into the fabric, see Figure 7 . It is evident that the fabric structure controls the spreading rate of a liquid droplet. The drop profile presents an important peak impact for the weft count. Therefore, this phenomenon is slightly faster for the less dense woven fabric. Then, spreading rate is very pronounced for the weak weft count. In addition, the two parameters characterising the porous substrate are its porosity and permeability. So, for this last fabric, the adimensional diameter variation shows greater spreading ratio which may be caused by the porosity where the drop penetration is much accentuated.

However, the spreading ratio is slow for the very dense fabric because the force acting on the water drop is stronger and possesses a large portion of liquid-air interface as well as the liquid-solid interface. These air bubbles are responsible for the first important deformation of the drop after impact. Then the diffusion is due to the absorption and pores penetration.

Therefore, the highest used weft count presents the important spreading rate, which indicates less penetration ratio and lengthier time taken to complete this phenomenon contrarily to the less used weft count as shown in the adimensional diameter and height variation in Figure 7.

\subsection{Effect of coating paste}

The effect of the coating paste was investigated. Therefore, the dispersive paste was applied to the cotton fabric. The same drop size was used to analyse the water drop impact of the treated and untreated cotton. Figure 8 shows the variation of the contact angle, the adimensional diameter, and the height at the time. It was shown that the characteristics of the fabric strongly depend on their construction parameters such as the type and fineness of filaments, yarn fineness, yarn density, and the type of weave. These characteristics have a strong influence on and, in many cases, control the wetting properties. First, the applied coating paste allows to surround the textile fibre and makes it more absorbent. Thus, the coating paste permits to decrease the porosity of the fabric by obstructing the macropores and mesopores. Therefore, the penetration of the liquid is lower than in each of uncoated fabric. This explanation 

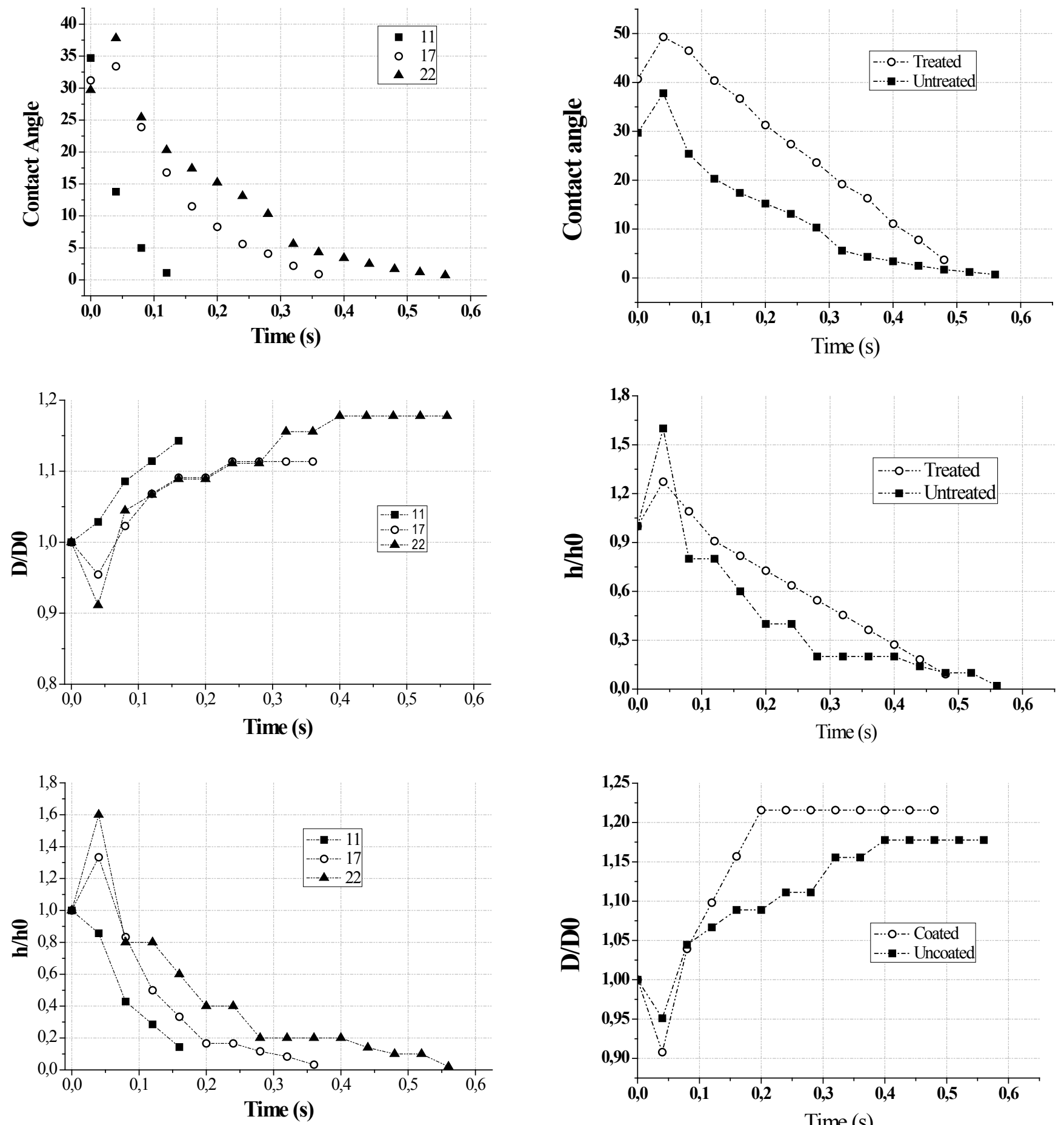

Figure 7. Drop impact parameters on woven cotton fabric

is proven by the important decrease of the adimensional height which confirms the fast penetration of the water drop.

Then, the spreading rate is very important for the treated fabric, as shown in the variation of D/D0 in Figure 8. The paste supports the spread of the water drop by increasing the hydrophilic character of the woven cotton fabric. Then, for the treated fabric, the energy of the drop is dissipated by viscous forces, and mostly converted into surface energy as the free surface area is greatly increased. Contrarily, the surface energy of the drop impact on uncoated fabric is very less, which explains its weak spreading rate.
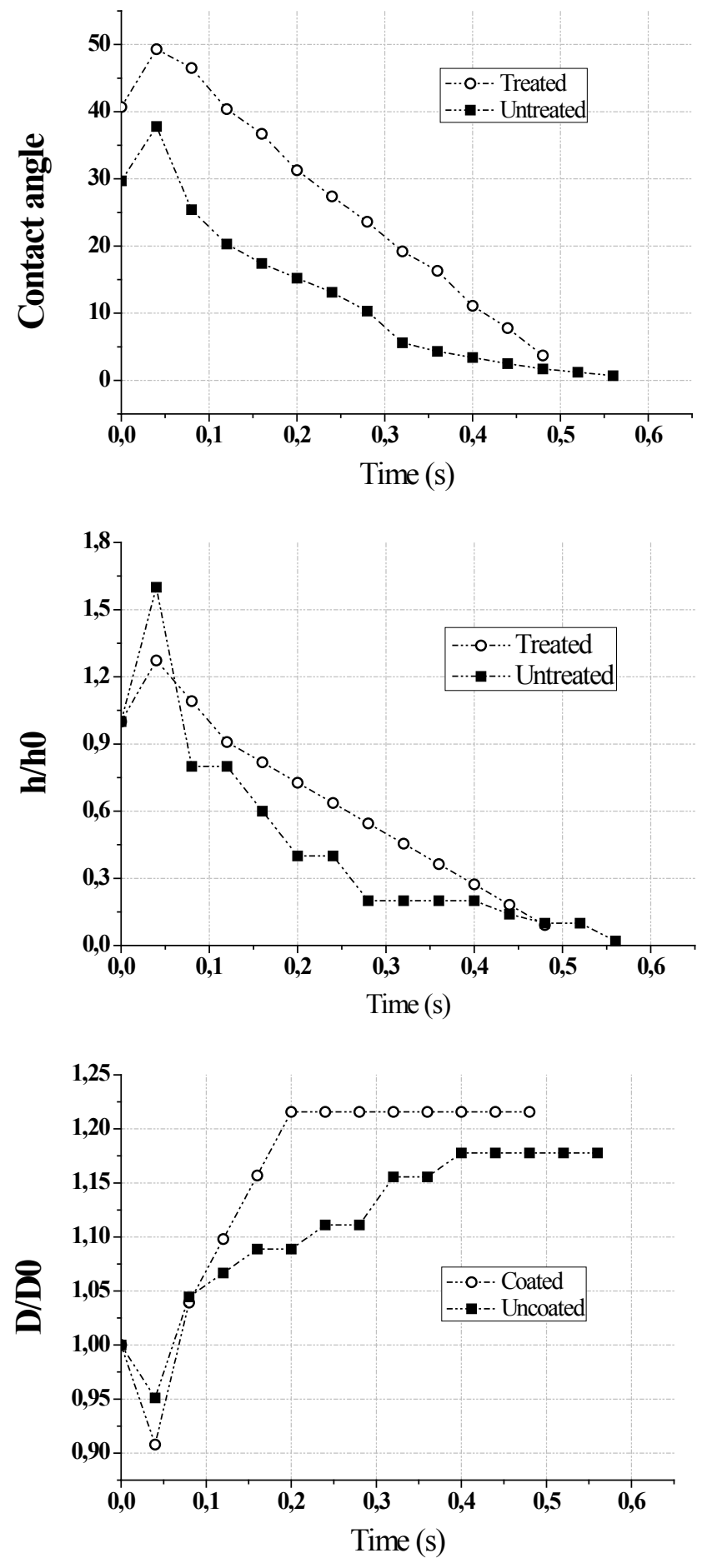

Figure 8. Drop impact parameters on coated woven cotton fabric

\section{Conlusions}

In summary, the drop impact on three substrates was studied and it is demonstrated that the drop shape behaves according to the energy of the impact. Therefore, three drop-spreading phases was identified and the drop perturbation in first short times was observed. This deformation is bound to the surface energy and it is much accentuated for the virgin glass and for the important weft count of cotton fabric. Thus, the drop profile is affected by the fabric porosity and it presents small deformation at first time compared to the virgin glass and the coating film. Then, the surface properties influence deeply 
the drop shape and the spreading rate. Moreover, the coating paste encourages spreading of the water drop by increasing the hydrophilic character of the woven cotton fabric, because the energy of the drop is dissipated by the viscous forces and converted into surface energy, which increases the spreading rate as the free surface area is greatly increased.

\section{Acknowledgements}

I would like to thank Ayda BAFFOUN, teacher in E.N.I.M, Monastir National School of Engineering, Tunisia, for the valuable assistance.

\section{References}

[1] Cassie, A.B.D.(1948). Contact Angles. Faraday Discussions, 3, 11-16.

[2] De Gennes, P.G. (1986). Deposition of Langmuir-Blodgett layers. Journal of Colloid Polymer and Science, 264(5), 463-465.

[3] Gillespie, T.(1958). The spreading of low vapor pressure liquids in paper, Journal of Colloid Interface and Science, 13, 32-50.

[4] Good, R.J. (1952). A Thermodynamic Derivation of Wenzel's Modification of Young's Equation for Contact Angles; Together with a Theory of Hysteresis, Journal of the American Chemical Society, 74(20), 5041-5042.

[5] Karbowiak, T.,Debeaufort,F., Champion,D., Voilley,A. (2006). Wetting properties at the surface of iotacarrageenan-based edible films. Journal of Colloid Interface and Science, 294(2), 400-410.

[6] Leidenfrost, J. G. (1966). On the fixation of water in diverse fire. International Journal of Heat and Mass Transfer, 9, 1153-1166.

[7] Marengo, M., Tropea, C. (1999). Aufprall von Tropfen auf Flüssigkeitsfilme. DFG, Zwischenbericht zum Forschungsvorhaben, Tr. 194/10.
[8] Mundo,C., Sommerfeld,M., Tropea, C. (1995). Droplet-wall collisions: Experimental studies of the deformation and breakup process. International Journal of Multiphase Flow, 21, 151-173.

[9] Mundo, C., Sommerfeld,M., Tropea, C. (1994). Experimental studies of the deposition and splashing of small liquid droplet impinging on a flat surface. Proceeding ICLASS, Rouen, France.

[10] Rioboo, R., Tropea, C., Marengo, M. (2001). Outcomes from a drop impact on solid surfaces. At. Sprays, 11, 155165.

[11] Scheller, B. L., Bousfield, D. W. (1995). Newtonian drop impact with a solid surface. Journal of Amerian Istitute of Chemical Engineers, 41(6), 1357-1367.

[12] Šikalo, Š., Marengo, M., Tropea, C., Ganic, E. N. (2002). Analysis of impact of droplets on horizontal surfaces. Experimental Thermal and Fluid Science, 25(7), 503-510.

[13] Stow, C. D., Hadfield, M. G. (1981). An experimental investigation of fluid flow resulting from the impact of a water drop with an unyielding dry surface. Proceeding of the Royal Society of London 373, 419-441.

[14] Vander Wal, R. L., Berger, G. M., Mozes, S. D. (2006). The splash/non-splash boundary upon a dry surface and thin fluid film. Experiments in Fluids, 40, 53-59.

[15] Washburn, E.W. (1921). The Dynamics of Capillary Flow. Physical Review 17 (3), 273-283.

[16] Wenzel, R.N. (1936). Resistance of solid surfaces to wetting by water. Industrial and Engineering Chemistry, 28, 988-994.

[17] Worthington, A. M. (1876). A second paper on the forms assumed by drop of liquids falling vertically on a horizontal plate. Proceeding of the Royal Society of London, 25, 489503..

[18] Young, T. (1805) An Essay on the cohesion of fluids. Philosophy Transactions of Royal Society of London Serie A, 95, 65-87.

[19] Zhang, X., Basaran, O. A. (1997). Dynamic surface tension effects in impact of a drop with a solid surface. Journal of Colloid Interface Science, 187(1), 166-178. 